\title{
THE SOURCE OF 3 MINUTE MAGNETOACOUSTIC OSCILLATIONS IN CORONAL FANS
}

\author{
D. B. Jess ${ }^{1}$, I. De Moortel ${ }^{2}$, M. Mathioudakis ${ }^{1}$, D. J. Christian ${ }^{3}$, K. P. Reardon ${ }^{1,4}$, P. H. Keys ${ }^{1}$, and F. P. Keenan ${ }^{1}$ \\ ${ }^{1}$ Astrophysics Research Centre, School of Mathematics and Physics, Queen’s University Belfast, Belfast BT7 1NN, UK; d.jess@qub.ac.uk \\ ${ }^{2}$ School of Mathematics and Statistics, University of St Andrews, St Andrews KY16 9SS, UK \\ ${ }^{3}$ Department of Physics and Astronomy, California State University Northridge, Northridge, CA 91330, USA \\ ${ }^{4}$ Osservatorio Astrofisico di Arcetri, I-50125 Firenze, Italy \\ Received 2012 July 2; accepted 2012 August 13; published 2012 September 13
}

\begin{abstract}
We use images of high spatial, spectral, and temporal resolution, obtained using both ground- and space-based instrumentation, to investigate the coupling between wave phenomena observed at numerous heights in the solar atmosphere. Analysis of $4170 \AA$ continuum images reveals small-scale umbral intensity enhancements, with diameters $\sim 0$ ' 6 , lasting in excess of 30 minutes. Intensity oscillations of $\approx 3$ minutes are observed to encompass these photospheric structures, with power at least three orders of magnitude higher than the surrounding umbra. Simultaneous chromospheric velocity and intensity time series reveal an $87^{\circ} \pm 8^{\circ}$ out-of-phase behavior, implying the presence of standing modes created as a result of partial wave reflection at the transition region boundary. We find a maximum waveguide inclination angle of $\approx 40^{\circ}$ between photospheric and chromospheric heights, combined with a radial expansion factor of $<76 \%$. An average blueshifted Doppler velocity of $\approx 1.5 \mathrm{~km} \mathrm{~s}^{-1}$, in addition to a time lag between photospheric and chromospheric oscillatory phenomena, confirms the presence of upwardly propagating slow-mode waves in the lower solar atmosphere. Propagating oscillations in EUV intensity are detected in simultaneous coronal fan structures, with a periodicity of $172 \pm 17 \mathrm{~s}$ and a propagation velocity of $45 \pm 7 \mathrm{~km} \mathrm{~s}^{-1}$. Numerical simulations reveal that the damping of the magnetoacoustic wave trains is dominated by thermal conduction. The coronal fans are seen to anchor into the photosphere in locations where large-amplitude umbral dot (UD) oscillations manifest. Derived kinetic temperature and emission measure time series display prominent outof-phase characteristics, and when combined with the previously established sub-sonic wave speeds, we conclude that the observed EUV waves are the coronal counterparts of the upwardly propagating magnetoacoustic slow modes detected in the lower solar atmosphere. Thus, for the first time, we reveal how the propagation of 3 minute magnetoacoustic waves in solar coronal structures is a direct result of amplitude enhancements occurring in photospheric UDs.
\end{abstract}

Key words: magnetohydrodynamics (MHD) - Sun: chromosphere - Sun: corona - Sun: oscillations - Sun: photosphere - sunspots

Online-only material: color figures

\section{INTRODUCTION}

Early white-light eclipse photographs of the Sun revealed elongated, faint columns of enhanced density stretching far out into the corona (van de Hulst 1950; Saito 1965). These structures, now commonly referred to as coronal plumes, can be viewed over a wide range of wavelengths, in particular the extreme-ultraviolet (EUV; Bohlin et al. 1975; Ahmad \& Withbroe 1977). Plumes are just one type of EUV feature that are seen to exist in the solar corona. Other examples include coronal loop and fan structures, which are observed to outline the coronal magnetic field topology, and demonstrate a wide range of oscillatory behavior (e.g., Aschwanden et al. 1999, 2002; Nakariakov et al. 1999; Jess et al. 2008b; Ofman \& Wang 2008; Verth et al. 2008; Van Doorsselaere et al. 2008; Ballai et al. 2011).

One of the first studies which uncovered propagating wave phenomena in coronal structures was that by Deforest \& Gurman (1998). These authors utilized the Extreme-ultraviolet Imaging Telescope on board the Solar and Heliospheric Observatory spacecraft to identify quasi-periodic perturbations in the brightness of $171 \AA$ A images. De Moortel et al. (2000) undertook a similar study using the higher spatial resolution Transition Region and Coronal Explorer (TRACE; Handy et al. 1999), and concluded that these oscillations were signatures of slow magnetoacoustic waves, which propagate upward along the coronal waveguides with velocities of $70-165 \mathrm{~km} \mathrm{~s}^{-1}$ and periods in the range 180-420 s. Energy estimates for these motions exhibit an incredibly wide range of values, typically $10^{2}-10^{5} \mathrm{erg} \mathrm{cm}^{-2} \mathrm{~s}^{-1}$ (Deforest \& Gurman 1998; De Moortel et al. 2000).

Since magnetic fields play an essential role in plume/fan/ coronal-loop formation and structuring, they are often modeled using magnetohydrodynamic (MHD) equations (Del Zanna et al. 1997). Utilizing nonlinear, two-dimensional MHD simulations, Ofman et al. (1999) were able to replicate previous observational results, and concluded that outward-propagating slow magnetoacoustic waves may be able to contribute significantly to the heating of the lower corona through compressive dissipation. Furthermore, theoretical modeling has suggested that the propagation characteristics of a magnetoacoustic intensity perturbation depends on a number of factors, including the dissipation of the wave energy (Klimchuk et al. 2004; De Moortel \& Nakariakov 2012). Numerical simulations indicate that thermal conduction may be the dominant damping mechanism behind the dissipation of magnetoacoustic wave energy in the solar corona (Ofman \& Wang 2002; De Moortel \& Hood 2003, 2004; Mendoza-Briceño et al. 2004).

Through examination of coronal structures in the close proximity of active regions, Fludra (2001) and Marsh \& Walsh 
(2006) were able to reveal how structures situated above sunspot regions displayed intensity oscillations with a period of the order of 3 minutes, while oscillations in "non-sunspot" structures demonstrated much longer periodicities (De Moortel et al. 2002). The authors concluded that the most likely explanation for the observed longitudinal waves revolves around a driver directly exciting the magnetic footpoints. This scenario requires the magnetoacoustic wave trains to be able to propagate from the lower solar atmosphere, through the transition region, and into the corona. Utilizing numerical simulations of the Sun's lower atmosphere, Khomenko \& Collados (2006) have revealed how longitudinal oscillations, generated with a periodicity of $\sim 3$ minutes in sunspot umbrae, can readily propagate upward from the photosphere and into the chromosphere.

Previously, 3 minute umbral oscillations have been notoriously difficult to detect at photospheric heights. Balthasar et al. (1987) were unable to detect any photospheric signatures using the Locarno solar station at the Göttingen Observatory, while Lites \& Thomas (1985) suggested that they may get lost in the noise, as a result of their very low amplitude. Indeed, Nagashima et al. (2007) utilized Hinode/SOT image sequences to show how oscillatory power, at all frequencies, is significantly reduced in sunspot umbrae. More recently, Kobanov et al. (2008, 2011a) have not only detected photospheric 3 minute oscillations, but the authors also claim that the location of maximum chromospheric power also corresponds to a co-spatial decrease in power of the photospheric oscillations. However, the spatial resolution obtained by Kobanov et al. (2008, 2011a) was on the order of $1^{\prime \prime}$, so precise diagnostics of the exact umbral structures displaying 3 minute periodicities was impossible.

In this paper, we utilize ground- and space-based instrumentation, with high spatial, temporal and spectral resolution, to investigate the origin of 3 minute magnetoacoustic waves observed in EUV images of coronal fan structures. We employ a multi-wavelength approach to study the photospheric counterpart of these coronal phenomena, and analyze the resulting wave propagation characteristics from the photosphere, through the chromosphere, and out into the corona.

\section{OBSERVATIONS AND DATA PREPARATION}

\subsection{Ground-based Data}

The observational data presented here are part of a sequence obtained during 13:32-14:04 UT on 2011 July 13, with the Dunn Solar Telescope (DST) at Sacramento Peak, New Mexico. We employed the Rapid Oscillations in the Solar Atmosphere (ROSA; Jess et al. 2010a) camera system to image a $69^{\prime \prime} \times 69^{\prime \prime}$ region encompassing active region NOAA 11250, positioned at heliocentric coordinates $\left(-146^{\prime \prime},-486^{\prime \prime}\right)$, or S26.3E10.1 in the conventional north-south-east-west coordinate system. A spatial sampling of 0 '.069 per pixel was used for the ROSA cameras, to match the telescope's diffraction-limited resolution in the blue continuum to that of the CCD. This results in images obtained at longer wavelengths being slightly oversampled. However, this was deemed desirable to keep the dimensions of the field of view the same for all ROSA cameras.

A recent addition to the DST's imaging capabilities is a new high quantum efficiency device, the Hydrogen-Alpha Rapid Dynamics camera (HARDcam). This camera, an iXon X3 DU-887-BV ${ }^{5}$ model manufactured by Andor Technology,

\footnotetext{
5 Full specifications available at http://www.andor.com.
}

Table 1

ROSA/HARDcam/IBIS Filter and Cadence Overview

\begin{tabular}{lccc}
\hline \hline Filter Used & $\begin{array}{c}\text { Exposure Time } \\
(\mathrm{ms})\end{array}$ & $\begin{array}{c}\text { Frames per } \\
\text { Second }\end{array}$ & $\begin{array}{c}\text { Reconstructed } \\
\text { Cadence }(\mathrm{s})\end{array}$ \\
\hline Blue continuum $(4170 \AA)$ & 7 & 30.3 & 1.06 \\
H $\alpha$ core & 35 & 27.9 & 1.26 \\
Ca II $(8542.12 \AA)$ & 250 & $3.0^{\mathrm{a}}$ & $43.4^{\mathrm{b}}$ \\
\hline
\end{tabular}

Notes.

a Average frames per second including Fabry-Perot tuning time.

${ }^{\mathrm{b}}$ Cadence of a complete Ca II profile scan.

consists of a back-illuminated, $512 \times 512$ pixel $^{2}$ electronmultiplying CCD, with a quantum efficiency exceeding $95 \%$ at $6500 \AA$. As a result, this camera is best suited to imaging in the red portion of the optical spectrum. The triggering and readout architectures are identical to the first-generation ROSA instrumentation, allowing HARDcam to be seamlessly integrated with the existing setup. To optimize the exploitation of its high quantum efficiency, HARDcam was employed behind a $0.25 \AA$ $\mathrm{H} \alpha$ core filter, incorporating a spatial sampling of 0 '.138 per pixel, providing a field-of-view size $\left(71^{\prime \prime} \times 71^{\prime \prime}\right)$ comparable to existing ROSA image sequences.

In addition to ROSA and HARDcam observations, the Interferometric BIdimensional Spectrometer (IBIS; Cavallini 2006) was used to simultaneously sample the Ca II absorption profile at $8542.12 \AA$. IBIS employed a spatial sampling of 0 '.097 per pixel, which allowed ROSA's near square field of view to be contained within the circular aperture provided by IBIS. A total of 13 discreet wavelength steps, with 10 exposures per step to assist image reconstruction, were used to provide a complete scan cadence of $43.4 \mathrm{~s}$. A white-light camera, synchronized with the IBIS feed, was utilized to assist the processing of narrowband images. Full details of the observations presented here, including filters and exposure times used, can be found in Table 1, while sample images can be viewed in Figure 1.

During the observations, high-order adaptive optics (Rimmele 2004) were used to correct wave-front deformations in real time. The acquired images were further improved through speckle reconstruction algorithms (Wöger et al. 2008), utilizing $32 \rightarrow 1$ restorations for the G-band and $4170 \AA$ continuum images. The remaining HARDcam and IBIS images were processed with $35 \rightarrow 1$ and $10 \rightarrow 1$ restorations, respectively. Postreconstruction cadences are displayed in the fourth column of Table 1. A full image-reconstructed IBIS scan through the Ca II absorption line includes a blueshift correction, required due to the use of classical etalon mountings (Cauzzi et al. 2008). Atmospheric seeing conditions remained excellent throughout the time series. However, to ensure accurate co-alignment in all bandpasses, broadband time series were Fourier co-registered and de-stretched using a $40 \times 40$ grid, equating to $\mathrm{a} \approx 1^{\prime \prime}$.7 separation between spatial samples (Jess et al. 2007a, 2008a). Narrowband images, including those from IBIS, were corrected by applying de-stretching vectors established from simultaneous broadband reference images (Reardon \& Cavallini 2008; Jess et al. 2010b; Reardon et al. 2011).

\subsection{Space-based Data}

The Atmospheric Imaging Assembly (AIA; Lemen et al. 2011) onboard the Solar Dynamics Observatory (SDO; Pesnell et al. 2011) was utilized to provide simultaneous EUV images of active region NOAA 11250 . This instrument images the entire 

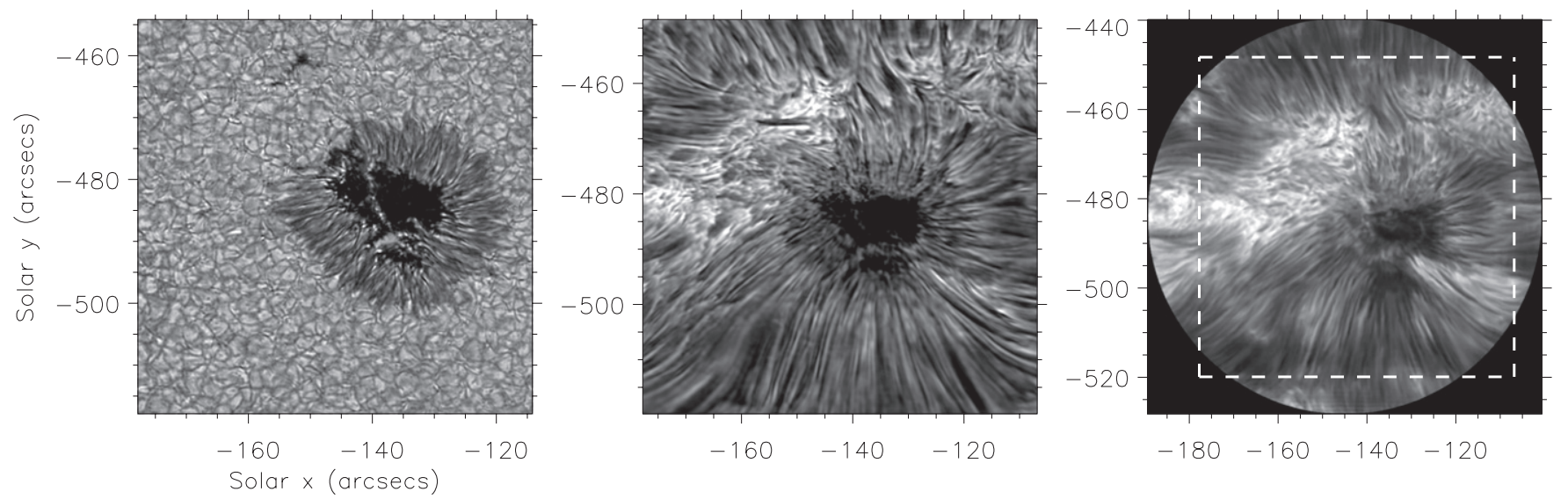

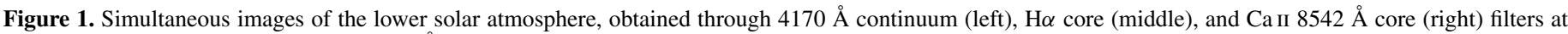

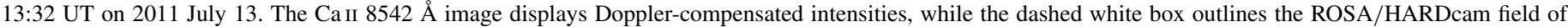
view. Axes are in heliocentric arcseconds, where $1^{\prime \prime} \approx 725 \mathrm{~km}$ on the solar surface.

solar disk in 10 different channels, incorporating a two-pixel spatial resolution of 1".2, and a cadence of $12 \mathrm{~s}$. Here, we selected seven EUV data sets spanning 13:30-14:05 UT on 2011 July 13, consisting of 175 images in each of the $94 \AA, 131 \AA$, $171 \AA$, $193 \AA, 211 \AA, 304 \AA$, and $335 \AA$ channels. In addition, one contextual $4500 \AA$ continuum image, acquired at 14:00 UT, was obtained for the purposes of co-aligning AIA data sets with images of the lower solar atmosphere.

The EUV bandpasses were specifically chosen to cover a wide range of transition region and coronal temperatures, spanning approximately 50,000 K-7 MK, under nonflaring conditions. Transition region imaging is covered by the He II-dominated $304 \AA$ A bandpass, with a typical formation temperature of $\sim 50,000 \mathrm{~K}$ (Jess et al. 2008c). The selected coronal channels, consisting of the $94 \AA, 131 \AA, 171 \AA, 193 \AA, 211 \AA$, and $335 \AA$ bandpasses, demonstrate typical effective temperatures of approximately 7.0 MK, 0.4 MK, $0.7 \mathrm{MK}, 1.6 \mathrm{MK}$, 2.0 MK, and 2.8 MK, respectively (O'Dwyer et al. 2010; Brooks et al. 2011). Thus, the AIA data sets, in conjunction with our ground-based observations of the lower solar atmosphere, provide us with the ideal opportunity to investigate the coupling of lower atmospheric (photosphere and chromosphere) phenomena with their multi-million degree coronal counterparts, at unprecedented spatial and temporal resolutions.

The AIA data were processed using the standard aia_prep routine, and include the removal of energetic particle hits, in addition to the co-registration of images from different wavelengths on to a common plate scale. Subsequently, $200^{\prime \prime} \times$ $200^{\prime \prime}$ sub-fields were extracted from the processed data, with a central pointing close to that of the ground-based image sequences. A sub-field image, including an outline of the field of view obtained with ground-based observations, is shown in the upper-left panel of Figure 2. Using the AIA $4500 \AA$ context image to define absolute solar coordinates, our ground-based observations were subjected to cross-correlation techniques to provide sub-pixel co-alignment accuracy between the imaging sequences. To do this, the plate scales of our ground-based observations were first degraded to match that of the AIA image. ${ }^{6}$ Next, squared mean absolute deviations were calculated between the data sets, with the ground-based images subsequently shifted to best align with the AIA reference image (see the lower panels of Figure 2 for interlaced examples).

\footnotetext{
6 Data analysis was performed on full-resolution (i.e., non-degraded) image sequences.
}

Following co-alignment, the maximum $x$ - and $y$-displacements are both less than one-tenth of an AIA pixel, or 0'.06 $(\approx 45 \mathrm{~km})$.

\section{DATA ANALYSIS AND DISCUSSION}

Using a combination of ground- and space-based data sets, we were able to analyze image sequences from the photosphere through to the corona. Due to the wide range of atmospheric heights covered, this section is divided into sub-sections in which we discuss different regions of the solar atmosphere.

\subsection{Photosphere}

To investigate oscillatory phenomena occurring in the sunspot umbra, we first masked out all other areas of the field of view. This was deemed desirable so that intrinsically faint umbral structures would become much more apparent in scaled intensity images. To isolate the umbra, we first had to create a binary map, whereby umbral pixels were given a value of " 1 ", and nonumbral pixels assigned a value of " 0 ." This form of binary map was created by first averaging the $4170 \AA$ A continuum images over the entire 32 minute duration of the data set. Next, the umbral pixels were defined as those with an intensity below $45 \%$ of the median granulation intensity, and subsequently assigned a value of " 1 ." This accurately defined the perimeter of the dark umbra. However, some small-scale structures, which existed inside the umbra (e.g., umbral dots (UDs)), exhibited higher relative intensity values. Therefore, to include all structures which existed inside the umbral boundary, all pixels which lay inside the outer perimeter were assigned a value of " 1 ," while all other pixels were given a value of " 0. ." The time-averaged $4170 \AA$ A continuum image, including the outline of the umbral binary map, is shown in the left panel of Figure 3.

Examination of the time-averaged umbral intensity map (middle panel of Figure 3) revealed a collection of nearcircular brightenings. These brightenings are consistent with the signatures of UDs, whereby they typically exhibit diameters between 0.2 and 0.8 , and intensities 1.3-1.5 times brighter than the background umbral value (Denker 1998; Tritschler \& Schmidt 2002; Sobotka \& Hanslmeier 2005). Due to their sharp appearance in a time-averaged intensity image, it hints at the persistent nature of such structures in the same spatial location, either through long lifetimes (e.g., 30 minutes or more; Sobotka et al. 1997a, 1997b), or by the reappearance of new UDs in the same spatial location. Analyzing simultaneous G-band and continuum filtergrams, Rimmele (2008) found that UDs, when 

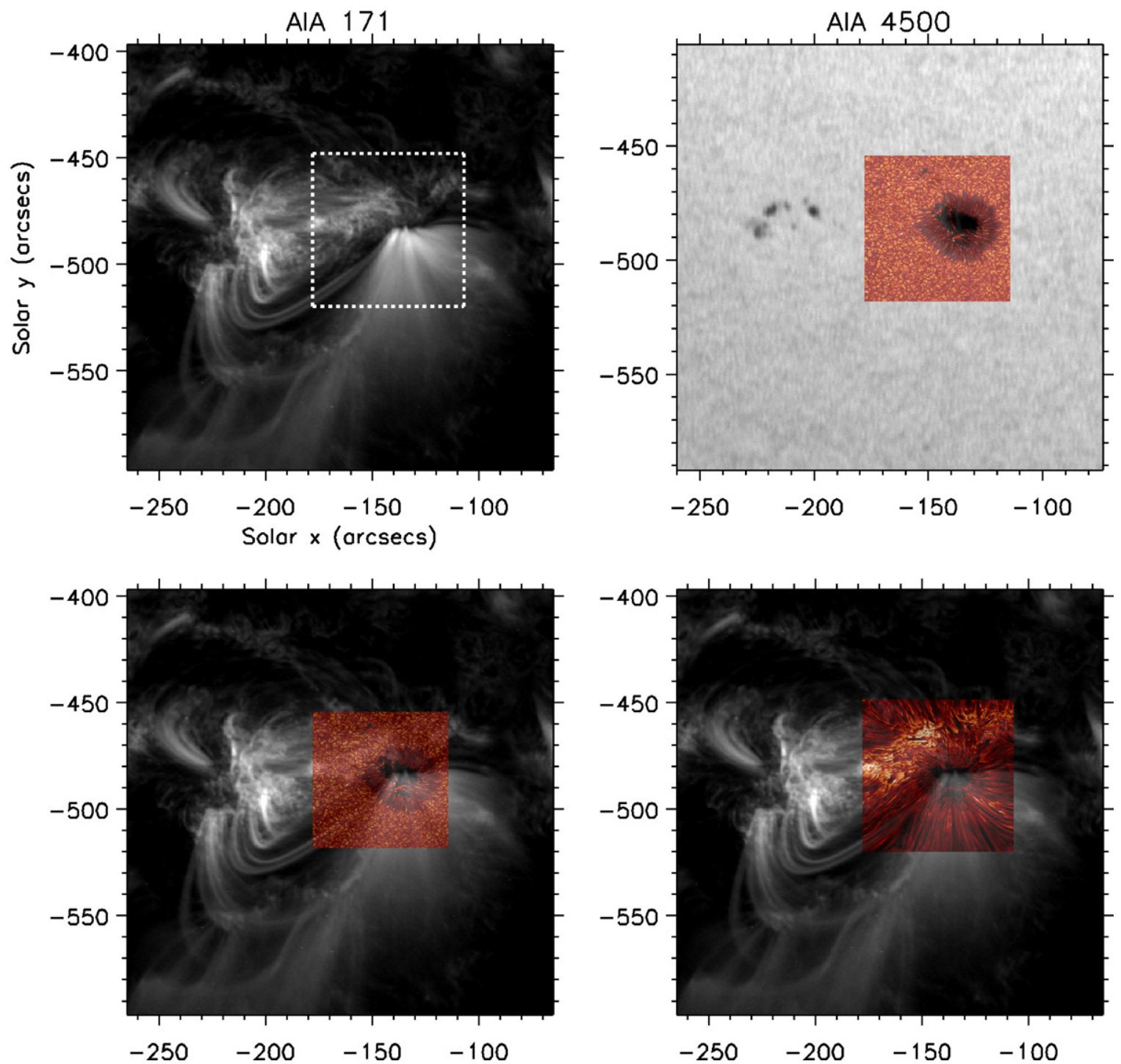

Figure 2. Top left: an AIA $171 \AA$ A image, taken at 13:32 UT, including a dashed white box to indicate the ROSA/HARDcam field of view. Top right: an AIA $4500 \AA$ context image, interlaced with a ROSA $4170 \AA$ A continuum snapshot, following sub-pixel co-alignment. Bottom panels: excellent co-alignment between lower and upper solar atmosphere data sets allow for precise spatial diagnostics, as demonstrated by the interlacing of $4170 \AA$ continuum (left) and $\mathrm{H} \alpha$ core (right) images, with the coronal emission seen in the AIA 171 A bandpass.

(A color version of this figure is available in the online journal.)

observed in the $\mathrm{G}$ band, displayed dark fine-structuring toward their core. Contrarily, this author found that these dark features are not as readily visible in the continuum, and concluded this may be due to the reduced resolution of the longer wavelength and longer exposure images. However, our continuum images were obtained at a shorter wavelength than the G band (4170 $\AA$ versus $4305 \AA$ ), in addition to being captured with a much shorter exposure time ( $7 \mathrm{~ms}$ versus $20 \mathrm{~ms}$ ). Thus, we cannot attribute a lack of dark fine-scale structuring in the UDs observed in the $4170 \AA$ continuum to either reduced spatial resolution, or exposure-time-related smearing of the images. Jess et al. (2012) recently determined the contribution functions of the G-band and $4170 \AA$ continuum filter bandpasses used at the DST, and concluded that the blue continuum is formed at a height of $\sim 25 \mathrm{~km}$, while the $\mathrm{G}$ band is formed approximately $100 \mathrm{~km}$ higher. This atmospheric height separation between the two bandpasses, in addition to the appearance of diatomic $\mathrm{CH}$ molecules in the G-band images, may contribute to the presence of dark cores observed in G-band UDs.
Since we are concerned with the larger macro-scale fluctuations in umbral intensity, rather than the very small scale structuring (e.g., dark features within UDs) which may be close to, if not overlap with the telescope's diffraction limit, our photospheric umbral time series was created purely from consecutive $4170 \AA$ continuum images. These images were multiplied by the umbral binary map, producing a data set which could be analyzed with Fourier techniques. Following the methodology of Jess et al. (2007a, 2007b), the wavelet analysis routines of Torrence \& Compo (1998) were applied to the $4170 \AA$ continuum umbral time series to search for the presence of oscillatory behavior. Considerable oscillatory power was present throughout the sunspot umbra, with dominant peaks in the Fourier spectrum at 3 and 5 minutes. This is consistent with the generalized $p$-mode spectrum of sunspot oscillations (Lites et al. 1982; Lites 1984, 1986; Bogdan \& Judge 2006). However, when locations of high oscillatory power were examined, it became evident that the UDs displayed significant enhancements of wave amplitude, at periodicities of approximately 3 minutes. The middle 

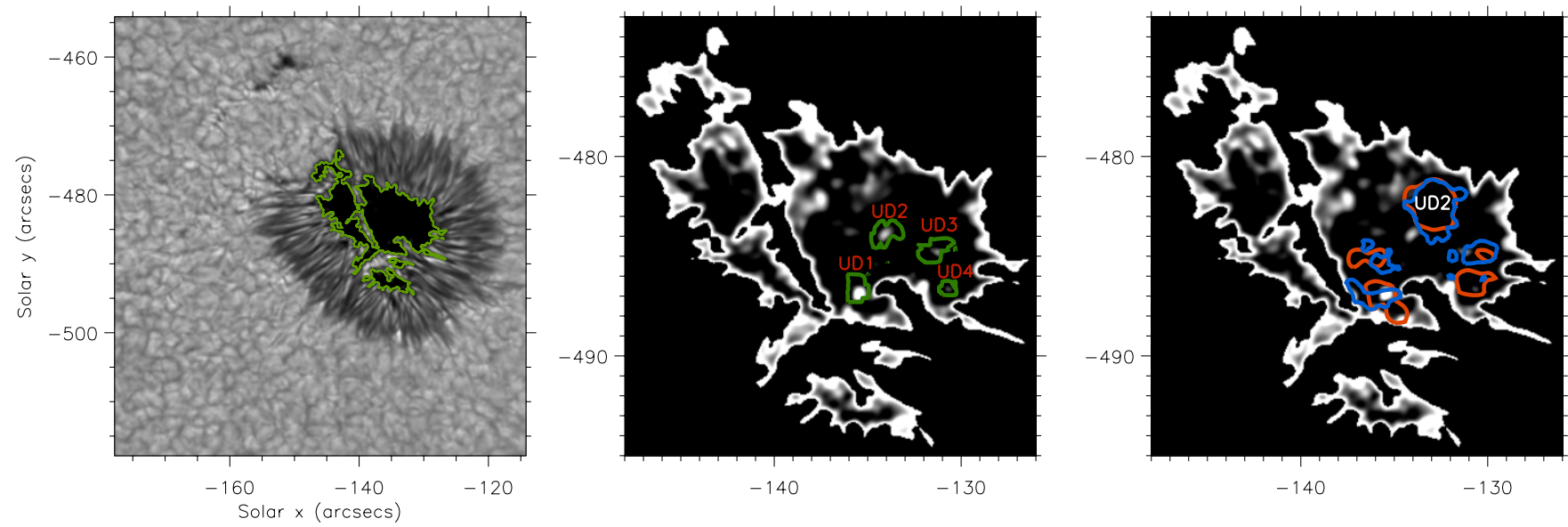

Figure 3. Left: a time-averaged 4170 A continuum image, where a solid green contour defines the outline of the sunspot umbra. Middle and right: a time-averaged close-up of the sunspot umbra, following isolation from the surrounding penumbral and granulation structures. Locations of the umbral dots analyzed here are indicated by red "UD $x$ " markings, where the " $x$ " refers to a number. Green, red, and blue contours indicate the locations where photospheric intensities, chromospheric $\mathrm{H} \alpha / \mathrm{Ca}$ II line core intensities, and Doppler velocities, respectively, display oscillatory power exceeding 1000 times the background umbral value, for a periodicity of approximately 3 minutes.

(A color version of this figure is available in the online journal.)
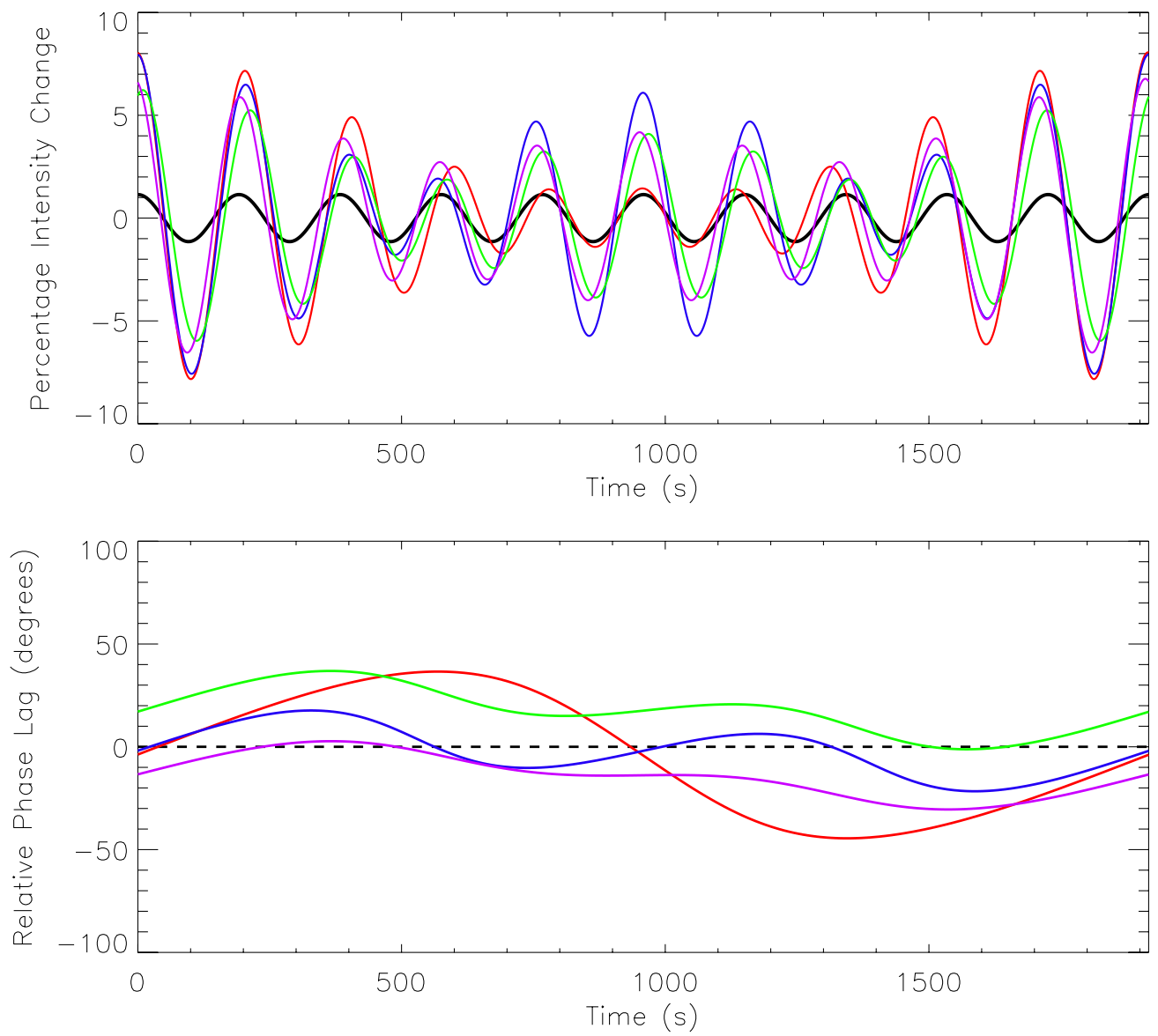

Figure 4. Upper: intensity time series of the four umbral dots labeled UD1 (red line), UD2 (blue line), UD3 (green line), and UD4 (pink line) in Figure 3, following temporal filtering using a 2-4 minute bandpass filter. The black line displays the intensity of the umbral region away from UD structures, devoid of long-lived or transient brightenings, and scaled (by a factor of five) for clarity. Lower: the relative phase angles between the 3 minute umbral dot and background umbral oscillations, plotted as a function of time. The red, blue, green, and pink lines represent umbral dots UD1, UD2, UD3, and UD4, respectively, while a black dashed line highlights a phase angle of $0^{\circ}$.

(A color version of this figure is available in the online journal.)

panel of Figure 3 shows the spatial locations where oscillatory power at approximately 3 minutes is in excess of 1000 times the background umbral value. To test whether these long-lived umbral brightenings oscillate in phase with the surrounding umbra, temporally filtered time series of these structures were first created by passing each spatially averaged UD intensity through a 2-4 minute bandpass filter. The resulting light curves, dominated by power corresponding to the 3 minute $p$-mode 
oscillations, are displayed in the upper panel of Figure 4, along with a filtered time series representing a region of the quiet umbra devoid of any transient brightenings. It is clear that not only do the peaks and troughs of the UD oscillations appear similar in time, but these signatures also closely follow the oscillations originating from within the background umbra.

To quantify any small-scale differences in the oscillating UD time series, a phase difference analysis with respect to the background umbra was performed. The lower panel of Figure 4 reveals the phase difference between each of the four UD time series, and an isolated region of the background umbra (i.e., the solid black line in the upper panel of Figure 4). It is clear that a preference exists for the UDs to oscillate in phase with the background umbra. Maximum deviations in the phase angle reach approximately $\pm 40^{\circ}$, although these may be due to slight drifts in oscillation period between the respective time series. For example, the phase angle related to UD1 (red line in the lower panel of Figure 4 ) drifts a total of $\approx 80^{\circ}$ over a $700 \mathrm{~s}$ time duration, providing an average shift of $\approx 0.11 \mathrm{~s}^{-1}$. This drift can be explained by a period discrepancy of only $20 \mathrm{~s}$ between the UD and the background umbra, something which is well within the normal range of $p$-mode frequencies (Thomas 1985). Thus, we interpret the umbra as a single oscillating "drum skin," which not only induces wave motion in its inherent UDs, but also causes these structures to oscillate in phase with one another.

While Chou et al. (2009) and Stangalini et al. (2011) have shown that 3 minute magnetoacoustic power is suppressed in sunspot umbrae as a result of local absorption and emissivity reduction, their spatial resolution was insufficient to allow studies of the smallest sunspot features (e.g., UDs). Recently, Shelyag et al. (2009) have shown that on small spatial scales the curvature and strength of magnetic field lines have substantial influence on the efficiency of magnetoacoustic wave propagation. Indeed, these authors suggest that wave power can actually be amplified under certain atmospheric conditions. Following on from this, our observational data suggest that UDs are able to enhance the background $p$-mode power by at least three orders of magnitude (see, e.g., middle panel of Figure 3). This may be a result of the magnetic field lines acting as efficient conduits for magnetoacoustic wave propagation (e.g., Singh 1992; Shelyag et al. 2006; Khomenko et al. 2008; Erdélyi \& Fedun 2010).

\subsection{Chromosphere}

Chromospheric information comes from the $\mathrm{H} \alpha$ core imaging data set, in addition to the Ca II $8542 \AA$ spectral imaging scans taken with IBIS. Furthermore, Doppler shifts of the Ca II profile minimum allow a series of two-dimensional velocity maps to be generated (Jess et al. 2010b). The Ca II core image shown in the right panel of Figure 1 is a true intensity map, created by establishing the line-profile minimum at each pixel. By displaying Doppler-compensated line-center intensities, rather than rest-wavelength intensities, brightness variations throughout the image are more indicative of the source function than of the velocities present in the line-forming region (Leenaarts et al. 2010).

In a process identical to that applied to the photospheric image sequence, the $\mathrm{H} \alpha$ and Doppler-compensated $\mathrm{Ca}$ II data sets were multiplied by the umbral binary map, and subsequently analyzed using wavelet techniques. As for the $4170 \AA$ continuum time series, considerable oscillatory power was present throughout the entire sunspot umbra, with dominant peaks in the Fourier spectrum at 3 and 5 minutes. However, remaining consistent with Thomas (1985), chromospheric power at periodicities of $\sim 5$ minutes was much reduced when compared to those found in the photospheric umbra. To investigate whether oscillatory power detected in the chromosphere can be related to similar phenomena found in the photosphere, the locations of high oscillatory power were examined. The right panel of Figure 3 outlines the spatial locations where power, in both intensity and velocity signals at approximately 3 minutes, is in excess of 1000 times the background umbral value. Similar to the locations of high photospheric power displayed in the middle panel of Figure 3, several distinct groups manifest in a horseshoe shape in the southwest quadrant of the sunspot umbra.

The locations of significant chromospheric power have two distinct differences when compared to their photospheric counterparts. First, their spatial positions are slightly offset from those displayed in the middle panel of Figure 3. Second, the spatial sizes of high chromospheric power are substantially larger than those found in the photosphere. These effects can be explained by the geometry of the magnetic field lines which stretch outward from the solar surface. For example, high photospheric power encompassing UD2 (middle panel of Figure 3) has a local maximum at the heliocentric coordinate $\left(-133^{\prime \prime} .7,-483^{\prime \prime} .7\right)$, while the chromospheric local maximum is at $\left(-133^{\prime \prime} .1,-482^{\prime \prime} .2\right)$. Thus, the offset between these two maxima is $\approx 1^{\prime \prime} .6$, or $\approx 1100 \mathrm{~km}$. A separation between the $4170 \AA$ continuum and the $\mathrm{H} \alpha / \mathrm{Ca}$ II core formation heights of $\sim 1800 \mathrm{~km}$ (Vernazza et al. 1981; Jess et al. 2012) requires an inclination angle of $\approx 40^{\circ}$ to support the assumption that the oscillatory signals are from a continuation of the same solar structure (e.g., Centeno et al. 2006). This is the largest offset present in our observations, and forms our upper limit of the magnetic field inclination angle. Rempel et al. (2009) have recently shown that the inclination angles (to the vertical) of magnetic flux tubes within sunspot umbrae can be quite large $\left(>45^{\circ}\right)$, with these structures often becoming horizontal near the penumbral boundary. Furthermore, Marsh et al. (2009) utilized stereoscopic observations of propagating slow-mode waves in coronal structures to infer an absolute inclination angle $\sim 40^{\circ}$. Thus, an inclination angle of $<40^{\circ}$ supports the interpretation that oscillatory behavior detected at photospheric and chromospheric layers are directly related by the magnetic field lines which extend upward from the solar surface.

An increase in the area of the oscillating regions can be associated with a physical expansion of the magnetic flux tubes as a function of atmospheric height. Through examination of intense magnetic field concentrations ( $>1000 \mathrm{G})$, Jess et al. (2009) were able to show that the radial expansion of magnetic flux tubes between photospheric and chromospheric heights can be as large as a factor of two. Assuming these magnetic flux tubes demonstrate an expanding cylindrical geometry, doubling the radial dimension will result in an area increase of $400 \%$. Continuing with the previous example, the region of high oscillatory power encompassing UD2 covers 326 pixels $\left(0.8 \mathrm{Mm}^{2}\right.$ at $2500 \mathrm{~km}^{2}$ pixel $\left.^{-1}\right)$ and 256 pixels $\left(2.5 \mathrm{Mm}^{2}\right.$ at $10,000 \mathrm{~km}^{2}$ pixel $^{-1}$ ) at photospheric and chromospheric heights, respectively. As a result, an expansion of only $310 \%$ is observed, meaning our observations are well within the limits of previous expanding magnetic flux tube models (e.g., Mein et al. 2007; Ruderman et al. 2008; Shelyag et al. 2010; Karami \& Bahari 2011; Fedun et al. 2011).

The specific mode of oscillation can be determined through investigation of the coupling between intensity and velocity signals. Here, we adopt the $V-I$ convention (Deubner \& Fleck 1989; Fleck \& Deubner 1989), which shows the delay 

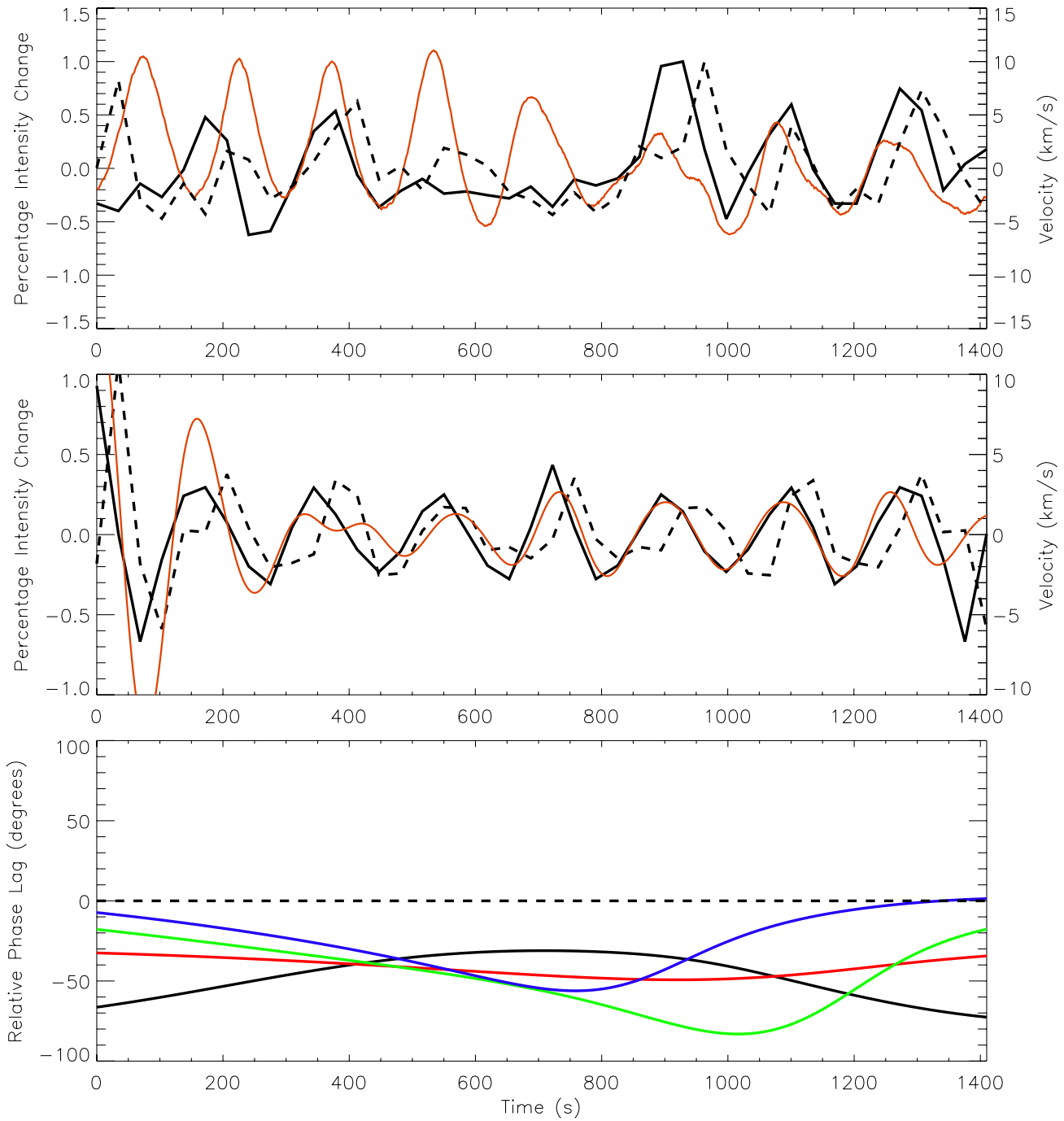

Figure 5. Upper: an unfiltered time series, created by spatially averaging Ca II intensity (solid line) and velocity (dashed line) signals, along with $\mathrm{H} \alpha$ core intensities (red line), originating from within the contours outlining "UD2" in the right panel of Figure 3. Middle: the same time series from the upper panel, following temporal filtering through a 2-4 minute bandpass filter. A $-90^{\circ}$ phase delay between velocity and intensity signals indicates the presence of oscillations which are magnetoacoustic in nature, while a close agreement between $\mathrm{H} \alpha$ and $\mathrm{Ca}$ II intensities is a result of their similar formation heights. Lower: the relative phase angles between the photospheric and chromospheric umbral dot oscillations, plotted as a function of time. The black, red, green, and blue lines represent umbral dots UD1, UD2, UD3, and UD4, respectively, while a black dashed line highlights a phase angle of $0^{\circ}$.

(A color version of this figure is available in the online journal.)

of maximum intensity $(I)$ with respect to maximum blueshift velocity $(V)$. Thus, a wave which has a velocity signal trailing its intensity signal by $1 / 4$ of a period will have a $V-I$ phase angle of $-90^{\circ}$. Due to the Ca II absorption line being sensitive to temperature fluctuations (Beebe \& Johnson 1969), we follow the common practice to adopt the intensity $(I)$ as a proxy for the local temperature. Mein (1977) has shown that when waves propagate along moderately inclined flux tubes, the phase lags between fluctuations in velocity and temperature are the same as those found in purely acoustic waves which have been modified by gravity. Therefore, in a fully adiabatic scenario, theory predicts a $V-I$ phase angle approaching $0^{\circ}$ for running acoustic waves, which can increase to $-90^{\circ}$ when aspects of wave reflection create standing acoustic modes (Hofmann et al. 1996; Al et al. 1998; Nigam \& Kosovichev 1999). Under isothermal conditions, the phase lag can further increase up to $-180^{\circ}$ (Mihalas \& Toomre 1981, 1982). Examination of the upper panel in Figure 5 reveals how the unfiltered velocity time series (dashed line) trails the co-spatial intensity light curve (solid line) by approximately $1 / 4$ of a wave period. This effect becomes even more pronounced when the same time series are passed through a 2-4 minute bandpass filter (middle panel of Figure 5). Chromospheric waves detected within the contours of the right panel of Figure 3 have a spatially and temporally averaged phase angle of $-87^{\circ} \pm 8^{\circ}$, suggesting these waves are magnetoacoustic in nature, with characteristics consistent with standing acoustic modes (Deubner 1974; Cram 1978). The generation of a chromospheric standing wave may be the result of a portion of the acoustic wave energy being reflected back at the transition region boundary (Schmitz \& Fleck 1992; Nakariakov et al. 2004; Fedun et al. 2011).

The spatial and temporal averaging of velocity signals found in the locations of high chromospheric power results in a net blueshift velocity of $\approx 1.5 \mathrm{~km} \mathrm{~s}^{-1}$ (upper panel of Figure 5). While a $V-I$ phase angle of $-87^{\circ} \pm 8^{\circ}$ implies the presence of wave reflection, a net blueshift velocity may also indicate that a portion of the magnetoacoustic wave is propagating in the upward direction. To examine the propagation characteristics of 
the magnetoacoustic waves associated with the UDs, spatially averaged $\mathrm{H} \alpha$ intensity time series were constructed for each of the regions contoured in the right panel of Figure 3, and subsequently passed through a 2-4 minute bandpass filter to isolate oscillatory phenomena around 3 minutes. A phase difference analysis was performed between these chromospheric $\mathrm{H} \alpha$ light curves and their corresponding photospheric $4170 \AA$ continuum counterparts created in Section 3.1, and displayed in the upper panel of Figure 4. A preference for negative phase angles is shown in the lower panel of Figure 5, which indicates the oscillatory signals are first observed in the photospheric $4170 \AA$ continuum, before later being detected in the chromospheric $\mathrm{H} \alpha$ time series. By averaging the derived phase angles over all UDs, an average phase lag of $-43^{\circ} \pm 12^{\circ}$ is found, implying the presence of upwardly propagating waves. Using the dominant periodicity of $\approx 3$ minutes, a relative phase angle of $-43^{\circ}$ equates to a time lag of $\approx 22 \mathrm{~s}$. This is consistent with the photosphere-to-chromosphere time-lag measurements detailed in Kobanov et al. (2011b). However, due to a height separation of $\sim 1800 \mathrm{~km}$ between the $4170 \AA$ continuum and $\mathrm{H} \alpha$ core image sequences (Vernazza et al. 1981; Jess et al. 2012), a time lag of $\approx 22 \mathrm{~s}$ requires a phase speed exceeding $80 \mathrm{~km} \mathrm{~s}^{-1}$. This is clearly unfeasible since a velocity this large will be supersonic, and violates our interpretation that the observed waves are magnetoacoustic slow modes. However, because the wave trains exist at the start of our observing sequence, and continue to be observed as the time series finishes, a factor of $n 2 \pi$ $\left(n 360^{\circ}\right)$, where $n$ is an integer, may be absent from our derived phase angle. By considering $n=1$, the phase lag increases to $\approx-403^{\circ}$, with the resulting phase speed reducing considerably to $\approx 8 \mathrm{~km} \mathrm{~s}^{-1}$. Only by observing the start and/or end of a propagating wave train can the exact time lag (and associated phase speed) be conclusively determined. Nevertheless, the detected waves are best described as upwardly propagating magnetoacoustic modes, which travel along magnetic flux tubes inclined to the vertical by less than $\approx 40^{\circ}$. Such an inclination angle may explain why Kobanov et al. (2008, 2011a) were unable to correlate (on a pixel-by-pixel basis) high chromospheric oscillatory power to that occurring in the underlying photosphere.

Our $\mathrm{H} \alpha$ data set has a much higher cadence than the Ca II core image sequence (1.26 s instead of $43.4 \mathrm{~s}$ ). Thus, examination of the $\mathrm{H} \alpha$ intensity time series can substantially reduce the associated errors when deriving the periodicities of the propagating magnetoacoustic waves. As can be seen in Figure 5, the $\mathrm{H} \alpha$ data display prominent intensity oscillations, most of which last for the entire duration of the time series. A spatial and temporal averaging over the regions encompassed by the red contours in the right panel of Figure 3 yields a peak chromospheric periodicity of $168 \pm 7 \mathrm{~s}$, consistent with the generalized $p$-mode spectrum of chromospheric sunspot oscillations (O'Shea et al. 2001, 2002; Banerjee et al. 2002). The close resemblance between $\mathrm{H} \alpha$ and $\mathrm{Ca}$ II intensity time series suggests their formation heights are remarkably similar. This is also apparent by the similarities present in simultaneous snapshots through their respective filters (e.g., Figure 1).

\subsection{Transition Region and Corona}

Due to the reduced spatial resolution of AIA images ( 2 pixel resolution $\approx 1^{\prime \prime} .2 \approx 870 \mathrm{~km}$ ), when compared with our simultaneous data sets of the lower solar atmosphere ( 2 pixel resolution $\approx 0$ '. $138 \approx 100 \mathrm{~km}$ ), it is imperative to concentrate on the larger scale structures which will be apparent in all image sequences. Following inspection of the co-aligned images displayed in the lower panels of Figure 2, it is clear that a number of fan structures rise out of the photospheric sunspot umbra, and into the corona. These structures are particularly visible as intensity enhancements in the coronal AIA images, where the $131 \AA, 171 \AA$, $193 \AA, 211 \AA$, and $335 \AA$ bandpasses show similar structuring extending outward in the southwest direction. However, these fan structures are mostly absent in the transition region $304 \AA$ images.

To investigate whether an absence of the fan brightenings in the $304 \AA$ images is a consequence of the structures lying outside of the filter's temperature response curve, differential emission measure (DEM) techniques were employed (e.g., Hannah \& Kontar 2012). Utilizing the six coronal EUV channels on AIA, we were able to construct emission measure (EM) and temperature $\left(T_{e}\right)$ estimates of active region NOAA 11250, including its immediate vicinity. Following the methodology presented by Aschwanden et al. (2011), each batch of near-simultaneous EUV exposures allowed us to construct a time series of EM and $T_{e}$ variables, thus allowing the evolutionary changes in each sequence to be studied. Importantly, the temperature of the coronal fan structures currently under investigation is in the range of 0.5-1.2 MK (right panel of Figure 6). Their relatively cool temperature, when compared to the multi-million degree values found at the center of the active region, probably manifest as a result of open magnetic field lines which cannot trap heated plasma. An alternative explanation could revolve around their connection with quasi-separatrix layers, which may be subject to a peculiar heating function (Schrijver et al. 2010). While the $304 \AA$ bandpass has two distinct temperature response functions covering approximately $50,000 \mathrm{~K}$ and $1.5 \mathrm{MK}$, the fact that active region NOAA 11250 was positioned close to solar disk center suggests that the resulting images should be dominated by the He II emission formed at $\sim 50,000 \mathrm{~K}$, with contributions from the 1.5 MK Si XI 202.22 $\AA$ emission line minimal (O'Dwyer et al. 2010). This helps to explain why features near one million degrees (e.g., the fan structures extending outward from the underlying sunspot), are not readily apparent in the transition region data.

Examination of time-lapse movies of coronal EUV images revealed clear and distinctly periodic outflows along the coronal fans. To quantify the associated periodicities and flow velocities, a series of one-dimensional slits were placed along the motion path in each of the coronal EUV channels. The resulting time-distance cuts reveal numerous propagating wave fronts, indicated by straight, diagonal trends in the bottom panel of Figure 7. The AIA EUV images, in the temperature range of $0.4-2.8$ MK (incorporating the $131 \AA, 171 \AA, 193 \AA, 211 \AA$, and $335 \AA$ bandpasses), display a dominant periodicity of $172 \pm 17 \mathrm{~s}$, and a propagation velocity of $45 \pm 7 \mathrm{~km} \mathrm{~s}^{-1}$. Red and blue contours in the lower panel of Figure 7 outline the intensity signals present in the higher temperature $211 \AA$ and $335 \AA$ bandpasses, respectively. It is clear that not only do the periodicities of the wave fronts closely resemble one another in both space and time, but the intensity gradients (and hence wave speeds) are also similar. Propagating wave fronts are observed co-spatially, and simultaneously in all but the $94 \AA$ AIA bandpass. The signal to noise of the $94 \AA$ channel is too low to extract a time series of sufficient quality for analysis. This may be compounded by the fact that the fan structures demonstrate a temperature much lower than the $94 \AA$ channel's peak response.

A periodicity of $\approx 172 \mathrm{~s}$ and a propagation velocity $\approx 45 \mathrm{~km} \mathrm{~s}^{-1}$ are consistent with King et al. (2003), who 

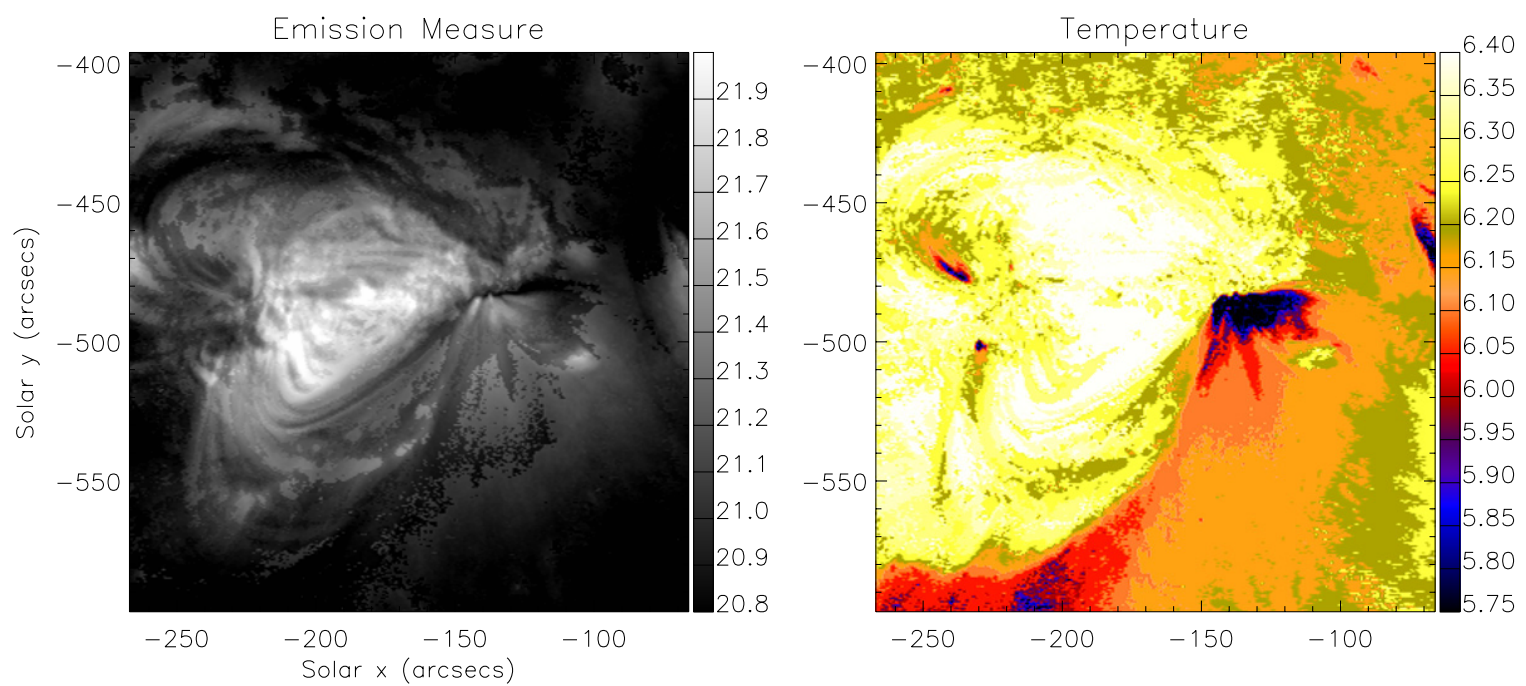

Figure 6. Two-dimensional emission measure (in units of $\mathrm{cm}^{-5} \mathrm{~K}^{-1}$; left) and temperature maps (in units of $\log \left(T_{e}\right)$; right), derived from near-simultaneous EUV snapshots of NOAA AR 11250. The coronal structures currently under investigation are located toward the southwest of the active region, where temperatures in the range of 0.6-1.2 MK dominate.

(A color version of this figure is available in the online journal.)

utilized the $171 \AA$ and $195 \AA$ filters onboard the TRACE satellite to reveal how the propagation velocity of such waves will not exceed the local sound speed, even with a large inclination angle of the waveguides away from the observer's line of sight. The typical sound speed associated with the dominant $\mathrm{Fe}$ IX emission from the $171 \AA$ A bandpass is $\approx 150 \mathrm{~km} \mathrm{~s}^{-1}$ (De Moortel 2006), which requires the fans currently under investigation to have an inclination angle exceeding $70^{\circ}$ before the observed wave motion would become supersonic. An inclination angle this large is highly unrealistic due to the location of the active region on the solar disk, in addition to previous surveys of coronal magnetic geometries (e.g., Aschwanden et al. 2002, 2008, 2009). Thus, we can conclude that the observed wave phenomenon is best described as propagating slow-mode waves.

Since propagating wave fronts are detected in many of the AIA EUV bandpasses, EM and temperature maps were subsequently investigated for the presence of similar signatures. As the EM maps created here are the sum of (squared) electron densities along a given line of sight, the total mass distribution, regardless of the local temperature, can be studied as a function of time (Aschwanden et al. 2011). Furthermore, the derived pixel-by-pixel temperatures correspond to the peak of the DEM distribution for a given line of sight, thus allowing the entire temperature range of the coronal plasma to be easily studied (Aschwanden \& Boerner 2011). Applying the same techniques used on the EUV image sequences, time-distance diagrams of the EM and $T_{e}$ variables derived for the fan structures reveal identical periodicities and propagation velocities as found in the EUV images (middle and upper panels of Figure 8). The EM is usually defined as (Kingston et al. 1982; Doyle et al. 1985)

$$
\operatorname{EM}\left(T_{e}\right)=\int n_{e}^{2} d h,
$$

where $n_{e}$ is the electron density and $h$ is an emitting depth along the observer's line of sight. Since the compressive phase of a magnetoacoustic wave mode will cause the emitting volume to decrease, thus reducing the overall EM, one would expect the EM time series to oscillate in phase with the corresponding EUV intensities. However, as the plasma becomes compressed, the associated kinetic temperature will increase, thus causing the $T_{e}$ signal to oscillate out of phase with the EM and EUV intensity signals. Indeed, perturbations in the EUV intensity, depicted in Figure 7, are found to occur in phase with the derived EM fluctuations. Furthermore, the lower panel in Figure 8 reveals how oscillations in $T_{e}$ are found to be $180^{\circ}$ out of phase with respect to those detected in the EM signal. Quantitatively, the detected fluctuations in the EM and $T_{e}$ values are typically in the range $21.50 \pm 0.03 \mathrm{~cm}^{-5} \mathrm{~K}^{-1}$ and $0.55 \pm 0.03 \mathrm{MK}$, respectively. This strengthens our interpretation that the observed wave phenomena are best described as magnetoacoustic slow modes.

\subsection{Numerical Simulations}

To model our observational findings, we performed a series of one-dimensional numerical simulations using the LagrangianRemap code (LARE; Arber et al. 2001), in which thermal conduction and optically thin radiation have been included (Owen \& De Moortel 2009). The one-dimensional model setup is constructed to closely match the observed AIA $171 \AA$ emission at the lowest coronal part of the fan structure. In particular, the density profile (upper-left panel of Figure 9) along the fan includes gravitational stratification, where the initial $(z=0)$ value is determined through equalization of the forward-modeled and observed $171 \AA$ intensities, as can be seen in the upper-right panel of Figure 9. Here, the solid line corresponds to the spatial variation in $171 \AA$ emission, obtained through forward-modeling of the temperature and density profiles inferred from the observed fan structure (where the forward modeling is undertaken using a code developed by De Moortel \& Bradshaw 2008). A model temperature profile is constructed which utilizes the minimum and maximum values of the (isothermal) temperatures extracted directly from the AIA emission. The background magnetic field is taken to be constant. From the upper-right panel of Figure 9, it is clear that the theoretical model is in close agreement with the observed $171 \AA$ emission during the first $15 \mathrm{Mm}$ along the fan. After this, the model emission declines more steeply than the observed $171 \AA$ emission. However, we are primarily concerned with the behavior within the first $15 \mathrm{Mm}$, and therefore can neglect 

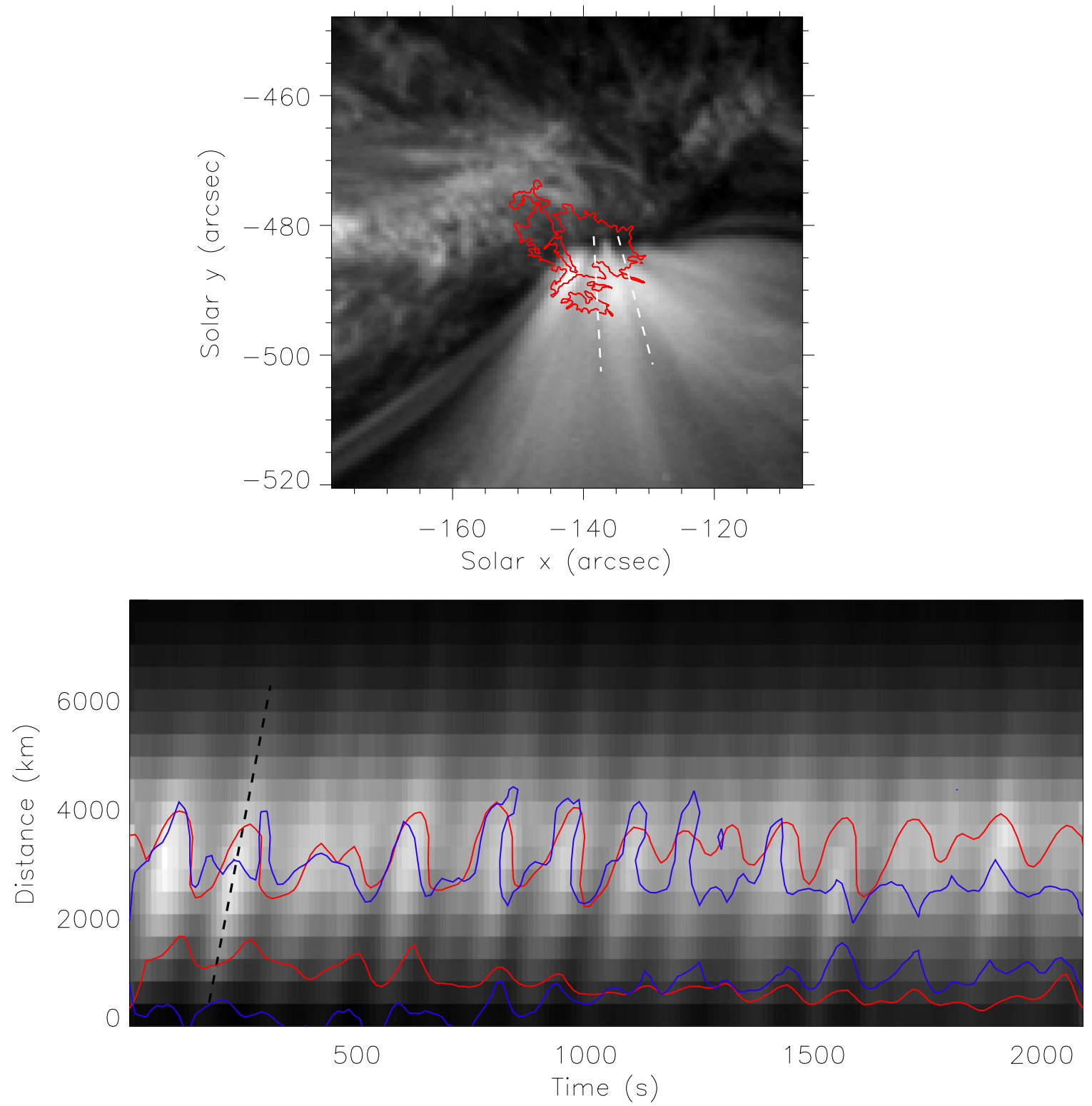

Figure 7. AIA $171 \AA \AA$ image (top), co-spatial with our field of view of the lower solar atmosphere. Dashed white lines outline a coronal fan structure, originating from within the underlying sunspot umbra, where propagating wave phenomena are readily apparent. The perimeter of the sunspot umbra is highlighted by a solid red line, demonstrating how the coronal fan is anchored into regions of high chromospheric oscillatory power (see, e.g., the right panel in Figure 3). A sample time-distance diagram of this fan is displayed in the lower panel, where $0 \mathrm{~km}$ represents the sunspot umbra. Red and blue contours outline $211 \AA$ and $335 \AA \AA$ intensities, respectively, which are $60 \%$ above the local quiescent background. A black dashed line represents the propagation of a typical wave front, where the gradient provides the wave speed, typically $45 \pm 7 \mathrm{~km} \mathrm{~s}^{-1}$. Wave phenomena which are co-spatial, co-temporal, and propagating with the same wave speed are readily apparent over a range of coronal temperatures.

(A color version of this figure is available in the online journal.)

larger distances where the emission becomes faint, and the observations become more affected by detector noise.

A slow magnetoacoustic wave is driven into the domain at the lower boundary, through periodic perturbations of the field-aligned velocity component. A snapshot of the velocity perturbations at $t=30$ minutes, using a driving period of $172 \mathrm{~s}$ to match that of the observations, is shown in the lower-left panel of Figure 9. A slow wave will propagate along the field at the characteristic tube speed, which is of the order of the local sound speed. Incorporating a temperature $\approx 1 \mathrm{MK}$, and a background magnetic field strength $\approx 10 \mathrm{G}$, the tube speed is of the order of $122 \mathrm{~km} \mathrm{~s}^{-1}$. Combined with a driving period of $172 \mathrm{~s}$, we expect a wavelength of the order of $22 \mathrm{Mm}$, which can be verified in the lower panels of Figure 9. After an initial increase, due to the rapidly decreasing background density, the velocity amplitudes are strongly damped through a combination of thermal conduction, optically thin radiation, and compressive viscosity. The corresponding density perturbations (i.e., the density profile at $t=0$ has been subtracted from the density at $t=30$ minutes) are shown in the lower-right panel of Figure 9. Such strong damping is predominantly caused by thermal conduction, as was previously uncovered by De Moortel $\& \operatorname{Hood}(2003,2004)$ and Owen \& De Moortel (2009). Only a small fraction of the overall damping can be attributed to optically thin radiation and compressive viscosity.

To achieve a more meaningful comparison with the perturbations observed by the AIA instrument, we utilized the model density and energy (temperature) distributions along the loop, 

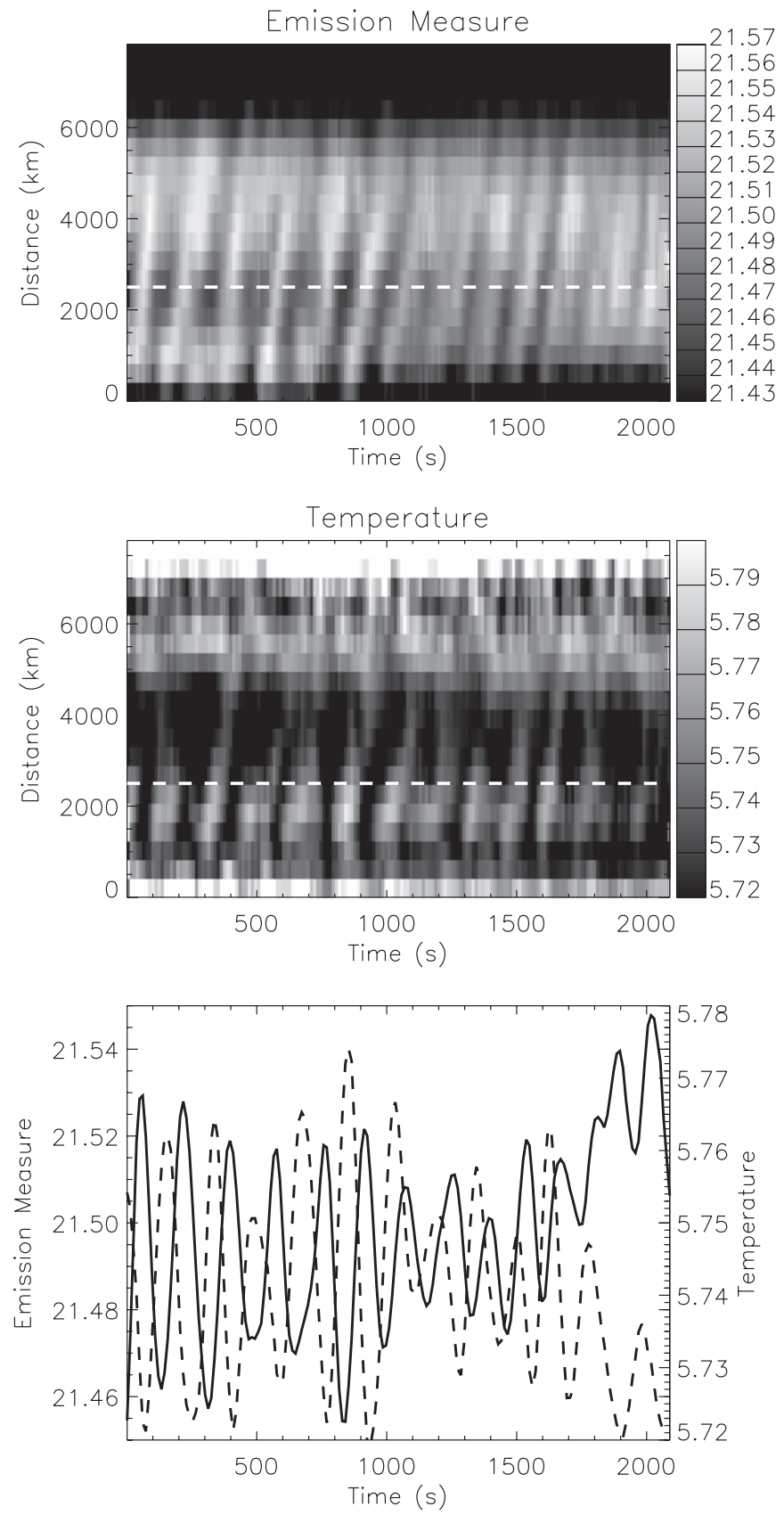

Figure 8. Time-distance diagrams of the emission measure (in units of $\mathrm{cm}^{-5} \mathrm{~K}^{-1}$; top) and temperature (in units of $\log \left(T_{e}\right)$; middle), derived for the fan structure where propagating wave phenomenon in EUV intensity images was detected. The dashed white lines highlight the spatial position along the slice ( $2500 \mathrm{~km}$ from the underlying umbra) where EM and $T_{e}$ time series were created. These values are displayed in the lower panel, where the solid line represents the emission measure, and the dashed line traces the temperature. Both time series are displayed in their native units, as used in the top and middle panels. A clear anti-correlation between the EM and $T_{e}$ time series is readily apparent.

to forward-model the emission for different AIA channels, at a multitude of time steps. We find that the modeled $171 \AA$ emission is significantly more intense over the first $15 \mathrm{Mm}$ of the coronal fan structure, when compared to the $193 \AA$ and $211 \AA$ intensities. Although these AIA channels contain some emission lines at cooler temperatures, the $193 \AA$ and $211 \AA$ bandpasses mainly respond to hotter coronal temperatures (i.e., above 1.5 MK; O’Dwyer et al. 2010; Brooks et al. 2011), and hence only begin to display elevated intensities when the density reduces and the local temperature increases beyond $1 \mathrm{MK}$. This is consistent with the AIA observations, whereby the cooler $171 \AA$ emission dominates the first $15 \mathrm{Mm}$ from the underlying sunspot. When forward-modeling intensity oscillations, it is important to remember that a variation in amplitude of the intensity perturbations is a combination of the change in amplitude of the model density and temperature fluctuations, as well as the individual response functions of the AIA channels to different temperatures.

Finally, we utilize the global wavelet power, at each spatial position along the coronal fan, to compare the amplitude decay rates found in the different AIA bandpasses in a more quantitative way. To do this, a wavelet transform was performed at each position along the fan, with the temporally averaged global wavelet power subsequently calculated. The red, green, and blue lines in Figure 10 represent the time-averaged oscillatory power around 3 minutes, for the AIA $171 \AA$, $193 \AA$, and $211 \AA$ observed and forward-modeled bandpasses, respectively. Note that each of these has been normalized to their individual maxima for display purposes. Of particular note is the observed $193 \AA$ power spectrum. Here, the solid green line represents the oscillatory power registered through the entire AIA bandpass, while the dashed green lines relate to the power associated with the hot $(\sim 1.6 \mathrm{MK})$ and cool $(\lesssim 1 \mathrm{MK})$ spectral components of this channel. These components have been separated using the methods detailed in Kiddie et al. (2012), with the resulting cool emission peaking alongside the $171 \AA$ power at $\approx 4.5 \mathrm{Mm}$, and the hot emission displaying a peak between the $171 \AA$ and $211 \AA$ bandpasses $(\approx 3 \mathrm{Mm})$. In reality, the global wavelet power of the $193 \AA$ and $211 \AA$ emission is substantially lower than that of the $171 \AA$ emission. However, the time-averaged power of the $193 \AA$ and $211 \AA$ modeled intensities are very similar, as is the case for the observations. For these wavelengths, the simulated global wavelet power reaches a minimum around $10 \mathrm{Mm}$, while the $171 \AA$ bandpass reaches its minimum power slightly further along the fan structure, at about $15 \mathrm{Mm}$. These results agree qualitatively with the observed time-averaged power, where the $193 \AA$ and $211 \AA$ channels near-simultaneously reach a minimum at about $9 \mathrm{Mm}$ along the coronal fan, with the $171 \AA$ power diminishing completely by $15 \mathrm{Mm}$. This reiterates the importance of thermal conduction in the damping of coronal slow-mode waves. The increase in time-averaged global wavelet power, after $\approx 10 \mathrm{Mm}$ in both the simulated and observed $193 \AA$ and $211 \AA$ time series, is due to the fact that, despite the decreasing amplitudes of the model density and temperature, the percentage intensity perturbations in these channels are actually increasing. This is a result of the increased sensitivity of the AIA $193 \AA$ and $211 \AA$ bandpasses to the $>1$ MK temperatures which have been reached by this distance along the fan structure. The increase is not as apparent in the observed $193 \AA$ and $211 \AA$ Aime-averaged wavelet power spectra, which may be due to the decreased signal-to-noise levels found in these channels, especially when compared to the $171 \AA$ A bandpass.

\section{OVERVIEW AND CONCLUDING REMARKS}

Here, we present high-cadence observations of the solar atmosphere, obtained using the latest ground- and space-based facilities. Prominent oscillatory behavior is detected throughout the optical and EUV image sequences, with remarkable similarities found between the detected wave modes. First, a number of UD structures in the photospheric umbra are found to display intensity oscillations with $\mathrm{a} \approx 3$ minute periodicity. These 

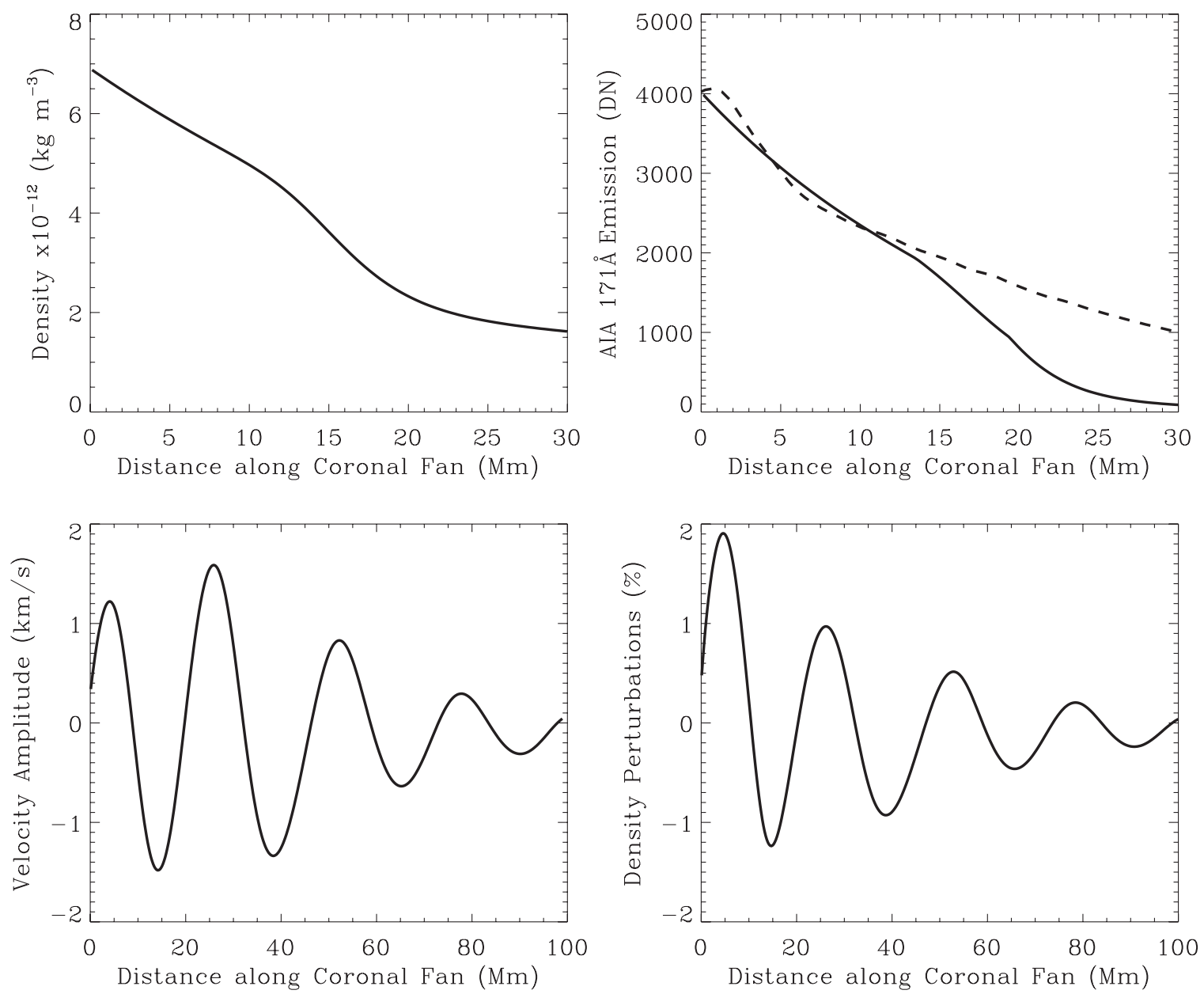

Figure 9. Initial (equilibrium) model density (upper left) used in our one-dimensional LARE simulations, displayed as a function of distance along the coronal fan. The forward-modeled AIA $171 \AA$ A emission (in DN pixel ${ }^{-1}$; solid line) is plotted in the upper-right panel, alongside the actual observed intensity profile (dashed line). Velocity amplitudes, induced by driving a slow magnetoacoustic wave into the numerical domain, are displayed in the lower-left panel, while the lower-right panel reveals the corresponding relative density perturbations, generated through subtraction of the forward-modeled values at $t=30$ minutes from those at the beginning of the simulation. Damping, caused primarily by thermal conduction, is readily apparent in the lower two panels. All horizontal axes are in units of Mm, where $1 \mathrm{Mm}=1000 \mathrm{~km}$.

oscillations exhibit considerable power, with regions encompassing the UDs displaying more than three orders of magnitude stronger power than the background umbra (Figure 3). Next, chromospheric intensity and velocity measurements were analyzed for the presence of co-spatial and co-temporal oscillations. Such phenomena were detected, both on larger spatial scales, and with small central offsets with respect to the underlying photospheric oscillations. By considering the extension of magnetic flux tubes from the solar surface out into the upper solar atmosphere, a geometric expansion of only $76 \%$ in the radial direction and an inclination angle $<40^{\circ}$ allows the observed oscillations to be interpreted as originating from within the same magnetic flux tube.

Following examination of the phase lag between chromospheric velocity and intensity components, a $V-I$ phase angle of $-87^{\circ} \pm 8^{\circ}$ was derived, allowing these waves to be described as a magnetoacoustic mode, with characteristics consistent with standing acoustic modes (middle panel of Figure 5). The generation of a chromospheric standing wave may be the result of partial wave reflection at the transition region boundary. An average blueshift velocity of $\approx 1.5 \mathrm{~km} \mathrm{~s}^{-1}$ was found in the locations where high chromospheric oscillatory power was present. This is a sub-sonic velocity, and coupled with a time lag between photospheric and chromospheric oscillatory phenomena, strengthens our interpretation that the observed oscillations are upwardly propagating magnetoacoustic waves, which originate in UD structures located in the photospheric umbra.

A prominent fan structure is present in the simultaneous coronal EUV images, namely, those from the AIA $131 \AA, 171 \AA$, $193 \AA, 211 \AA$, and $335 \AA$ bandpasses. This fan is not readily apparent in either the transition-region-dominated $304 \AA$ emission, or in the higher temperature ( 7.0 MK) $94 \AA$ bandpass. Using DEM techniques, we constrained the temperature of the coronal fan to $0.5-1.2 \mathrm{MK}$, thus placing it outside the temperature range of both the $94 \AA$ and $304 \AA$ filtergrams (Figure 6). Time-distance techniques were employed on the EUV imaging data where the fan structure was readily apparent, allowing the characteristics of propagating wave phenomena to be uncovered. Most coronal channels, regardless of their absolute temperature sensitivity, revealed outwardly propagating wave fronts with an average periodicity and velocity of $172 \pm 17 \mathrm{~s}$ and $45 \pm 7 \mathrm{~km} \mathrm{~s}^{-1}$, respectively (lower panel of Figure 7). The outof-phase nature between the derived temperature and EM signals indicates the presence of a compressive wave mode (Figure 8). This, coupled with a sub-sonic wave speed $\left(\approx 45 \mathrm{~km} \mathrm{~s}^{-1}\right)$, highlights the fact that these coronal phenomena are best described as upwardly propagating magnetoacoustic slow-mode waves. 

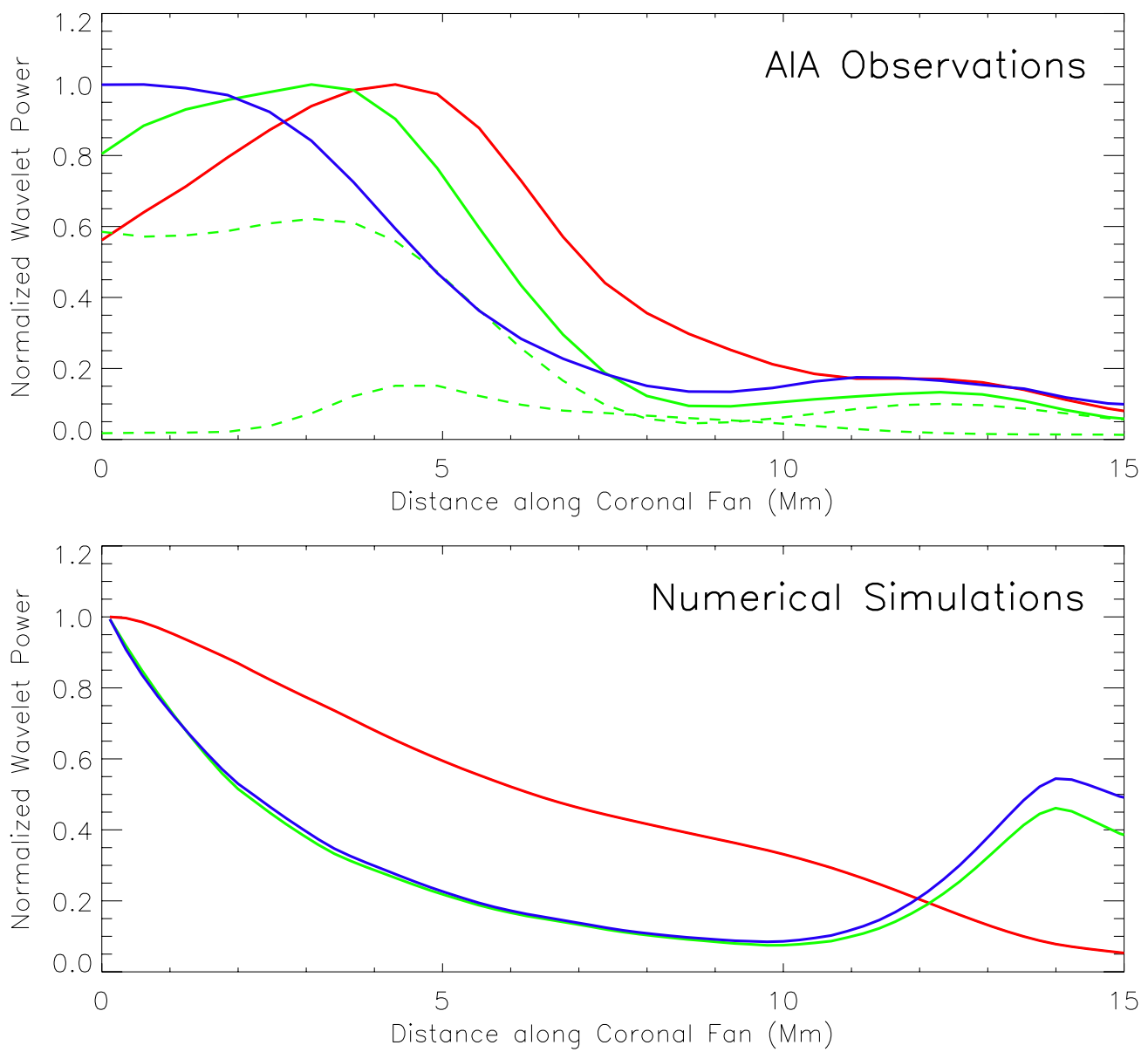

Figure 10. Time-averaged global wavelet power spectra, for the observed (top) and simulated (bottom) AIA time series, plotted as a function of distance along the coronal fan. The red, green, and blue lines highlight the oscillatory power contained within the $171 \AA, 193 \AA$, and $211 \AA$ A bandpasses, respectively, with each power spectrum normalized for clarity. Dashed green lines displayed in the observed spectra (top) relate to the power associated with the hot $(\sim 1.6 \mathrm{MK})$ and cool $(\leqslant 1 \mathrm{MK})$ spectral components of the $193 \AA$ channel, with the cool component closely following the $171 \AA$ trend, and the hot component tending more toward the $211 \AA$ spectrum. The damping of magnetoacoustic wave power, at hotter temperatures first, is an indication that thermal conduction is the dominant damping mechanism.

(A color version of this figure is available in the online journal.)

Employing numerical simulations, we were able to accurately simulate the behavior of the coronal EUV emission. Utilizing input parameters derived directly from the AIA observations, forward-modeling techniques allowed us to evolve velocity, density, and emissivity values forward in time, creating a time series which could be directly compared to the AIA observations. Crucially, our simulations revealed that thermal conduction is the primary damping mechanism behind the dissipation of magnetoacoustic slow-mode waves in the corona. Other mechanisms, including optically thin radiation and compressive viscosity, play a secondary role in the damping of these oscillations.

The fan structure observed in the AIA images, which displays signatures of propagating magnetoacoustic waves, appears to have anchor points in the southwest quadrant of the photospheric sunspot umbra (lower panels of Figure 2). These locations are also consistent with the presence of large-amplitude wave phenomena detected in simultaneous photospheric and chromospheric image sequences. The co-temporal and co-spatial relationship between these upwardly-propagating magnetoacoustic wave modes, detected throughout the entire solar atmosphere, suggests such coronal phenomena may be driven by UD oscillations occurring inside the sunspot umbra. With this conclusion, it appears that photospheric structures which are on the order of 0 .'5 $(360 \mathrm{~km})$ in diameter, can have a strong influence on coronal structures not only several thousand $\mathrm{km}$ above their position, but on structures which have expanded into the local plasma to diameters often exceeding 10" (7000 km).

D.B.J. thanks the Science and Technology Facilities Council (STFC) for a Post-Doctoral Fellowship. I.D.M. acknowledges support from a Royal Society University Research Fellowship. P.H.K. is grateful to the Northern Ireland Department of Education and Learning for a PhD studentship. D.J.C. thanks the CSUN College of Science for start-up funding related to this project. Solar Physics research at QUB is supported by STFC. The ROSA project is supported by The European Office of Aerospace Research \& Development. HARDcam observations were made possible by a Royal Society Research Grant (2009). We are grateful for the use of $S D O /$ AIA images, which were obtained courtesy of NASA/SDO and the AIA, EVE, and HMI science teams.

Facilities: Dunn (ROSA, HARDcam, IBIS), SDO (AIA)

\section{REFERENCES}

Ahmad, I. A., \& Withbroe, G. L. 1977, Sol. Phys., 53, 397

Al, N., Bendlin, C., \& Kneer, F. 1998, A\&A, 336, 743

Arber, T. D., Longbottom, A. W., Gerrard, C. L., \& Milne, A. M. 2001, J. Comput. Phys., 171, 151

Aschwanden, M. J., \& Boerner, P. 2011, ApJ, 732, 81 
Aschwanden, M. J., Boerner, P., Schrijver, C. J., \& Malanushenko, A. 2011 Sol. Phys., 384, 1

Aschwanden, M. J., de Pontieu, B., Schrijver, C. J., \& Title, A. M. 2002, Sol. Phys., 206, 99

Aschwanden, M. J., Fletcher, L., Schrijver, C. J., \& Alexander, D. 1999, ApJ, 520,880

Aschwanden, M. J., Wülser, J.-P., Nitta, N. V., \& Lemen, J. R. 2008, ApJ, 679, 827

Aschwanden, M. J., Wuelser, J.-P., Nitta, N. V., Lemen, J. R., \& Sandman, A. 2009, ApJ, 695, 12

Ballai, I., Jess, D. B., \& Douglas, M. 2011, A\&A, 534, A13

Balthasar, H., Wiehr, E., \& Kueveler, G. 1987, Sol. Phys., 112, 37

Banerjee, D., O’Shea, E., Goossens, M., Doyle, J. G., \& Poedts, S. 2002, A\&A, 395,263

Beebe, H. A., \& Johnson, H. R. 1969, Sol. Phys., 10, 79

Bogdan, T. J., \& Judge, P. G. 2006, Phil. Trans. R. Soc., 364, 313

Bohlin, J. D., Purcell, J. D., Sheeley, N. R., Jr., \& Tousey, R. 1975, BAAS, 7, 356

Brooks, D. H., Warren, H. P., \& Young, P. R. 2011, ApJ, 730, 85

Cauzzi, G., Reardon, K. P., Uitenbroek, H., et al. 2008, A\&A, 480, 515

Cavallini, F. 2006, Sol. Phys., 236, 415

Centeno, R., Collados, M., \& Trujillo Bueno, J. 2006, ApJ, 640, 1153

Chou, D.-Y., Yang, M.-H., Zhao, H., Liang, Z.-C., \& Sun, M.-T. 2009, ApJ, 706, 909

Cram, L. E. 1978, A\&A, 70, 345

De Moortel, I. 2006, Phil. Trans. R. Soc., 364, 461

De Moortel, I., \& Bradshaw, S. J. 2008, Sol. Phys., 252, 101

De Moortel, I., \& Hood, A. W. 2003, A\&A, 408, 755

De Moortel, I., \& Hood, A. W. 2004, A\&A, 415, 705

De Moortel, I., Ireland, J., Hood, A. W., \& Walsh, R. W. 2002, A\&A, 387, L13

De Moortel, I., Ireland, J., \& Walsh, R. W. 2000, A\&A, 355, L23

De Moortel, I., \& Nakariakov, V. M. 2012, Phil. Trans. R. Soc., 370, 3193

Deforest, C. E., \& Gurman, J. B. 1998, ApJ, 501, L217

Del Zanna, L., Hood, A. W., \& Longbottom, A. W. 1997, A\&A, 318, 963

Denker, C. 1998, Sol. Phys., 180, 81

Deubner, F.-L. 1974, Sol. Phys., 39, 31

Deubner, F.-L., \& Fleck, B. 1989, A\&A, 213, 423

Doyle, J. G., Mason, H. E., \& Vernazza, J. E. 1985, A\&A, 150, 69

Erdélyi, R., \& Fedun, V. 2010, Sol. Phys., 263, 63

Fedun, V., Shelyag, S., \& Erdélyi, R. 2011, ApJ, 727, 17

Fleck, B., \& Deubner, F.-L. 1989, A\&A, 224, 245

Fludra, A. 2001, A\&A, 368, 639

Handy, B. N., Acton, L. W., Kankelborg, C. C., et al. 1999, Sol. Phys., 187, 229

Hannah, I. G., \& Kontar, E. P. 2012, A\&A, 539, A146

Hofmann, J., Steffens, S., \& Deubner, F.-L. 1996, A\&A, 308, 192

Jess, D. B., Andić, A., Mathioudakis, M., Bloomfield, D. S., \& Keenan, F. P. 2007a, A\&A, 473, 943

Jess, D. B., Mathioudakis, M., Christian, D. J., et al. 2010a, Sol. Phys., 261, 363

Jess, D. B., Mathioudakis, M., Christian, D. J., Crockett, P. J., \& Keenan, F. P. 2010b, ApJ, 719, L134

Jess, D. B., Mathioudakis, M., Crockett, P. J., \& Keenan, F. P. 2008a, ApJ, 688, L119

Jess, D. B., Mathioudakis, M., Erdélyi, R., et al. 2008b, ApJ, 680, 1523

Jess, D. B., Mathioudakis, M., Erdélyi, R., et al. 2009, Science, 323, 1582

Jess, D. B., McAteer, R. T. J., Mathioudakis, M., et al. 2007b, A\&A, 476, 971

Jess, D. B., Rabin, D. M., Thomas, R. J., et al. 2008c, ApJ, 682, 1363

Jess, D. B., Shelyag, S., Mathioudakis, M., et al. 2012, ApJ, 746, 183

Karami, K., \& Bahari, K. 2011, Ap\&SS, 333, 463

Khomenko, E., \& Collados, M. 2006, ApJ, 653, 739

Khomenko, E., Collados, M., \& Felipe, T. 2008, Sol. Phys., 251, 589

Kiddie, G., De Moortel, I., Del Zanna, G., McIntosh, S. W., \& Whittaker, I. 2012, Sol. Phys., 279, 427

King, D. B., Nakariakov, V. M., Deluca, E. E., Golub, L., \& McClements, K. G. 2003, A\&A, 404, L1

Kingston, A. E., Doyle, J. G., Dufton, P. L., \& Gurman, J. B. 1982, Sol. Phys., 81,47

Klimchuk, J. A., Tanner, S. E. M., \& De Moortel, I. 2004, ApJ, 616, 1232
Kobanov, N. I., Kolobov, D. Y., \& Chupin, S. A. 2008, Astron. Lett., 34, 133

Kobanov, N. I., Kolobov, D. Y., Chupin, S. A., \& Nakariakov, V. M. 2011a, A\&A, 525, A41

Kobanov, N. I., Kustov, A. S., Chupin, S. A., \& Pulyaev, V. A. 2011b, Sol. Phys., 273,39

Leenaarts, J., Rutten, R. J., Reardon, K., Carlsson, M., \& Hansteen, V. 2010, ApJ, 709,1362

Lemen, J. R., Title, A. M., Akin, D. J., et al. 2012, Sol. Phys., 275, 17

Lites, B. W. 1984, ApJ, 277, 874

Lites, B. W. 1986, ApJ, 301, 992

Lites, B. W., \& Thomas, J. H. 1985, ApJ, 294, 682

Lites, B. W., White, O. R., \& Packman, D. 1982, ApJ, 253, 386

Marsh, M. S., \& Walsh, R. W. 2006, ApJ, 643, 540

Marsh, M. S., Walsh, R. W., \& Plunkett, S. 2009, ApJ, 697, 1674

Mein, N. 1977, Sol. Phys., 52, 283

Mein, P., Mein, N., Faurobert, M., Aulanier, G., \& Malherbe, J.-M. 2007, A\&A, 463, 727

Mendoza-Briceño, C. A., Erdélyi, R., \& Sigalotti, L. D. G. 2004, ApJ, 605, 493

Mihalas, B. W., \& Toomre, J. 1981, ApJ, 249, 349

Mihalas, B. W., \& Toomre, J. 1982, ApJ, 263, 386

Nagashima, K., Sekii, T., Kosovichev, A. G., et al. 2007, PASJ, 59, 631

Nakariakov, V. M., Ofman, L., Deluca, E. E., Roberts, B., \& Davila, J. M. 1999, Science, 285, 862

Nakariakov, V. M., Tsiklauri, D., Kelly, A., Arber, T. D., \& Aschwanden, M. J. 2004, A\&A, 414, L25

Nigam, R., \& Kosovichev, A. G. 1999, ApJ, 510, L149

O’Dwyer, B., Del Zanna, G., Mason, H. E., Weber, M. A., \& Tripathi, D. 2010, A\&A, 521, A21

Ofman, L., Nakariakov, V. M., \& Deforest, C. E. 1999, ApJ, 514, 441

Ofman, L., \& Wang, T. 2002, ApJ, 580, L85

Ofman, L., \& Wang, T. J. 2008, A\&A, 482, L9

O’Shea, E., Banerjee, D., Doyle, J. G., Fleck, B., \& Murtagh, F. 2001, A\&A, 368, 1095

O'Shea, E., Muglach, K., \& Fleck, B. 2002, A\&A, 387, 642

Owen, N., \& De Moortel, I. 2009, A\&A, 494, 339

Pesnell, W. D., Thompson, B. T., \& Chamberlin, P. C. 2011, Sol. Phys., 367

Reardon, K. P., \& Cavallini, F. 2008, A\&A, 481, 897

Reardon, K. P., Wang, Y.-M., Muglach, K., \& Warren, H. P. 2011, ApJ, 742,119

Rempel, M., Schüssler, M., Cameron, R. H., \& Knölker, M. 2009, Science, 325,171

Rimmele, T. R. 2004, Proc. SPIE, 5490, 34

Rimmele, T. 2008, ApJ, 672, 684

Ruderman, M. S., Verth, G., \& Erdélyi, R. 2008, ApJ, 686, 694

Saito, K. 1965, PASJ, 17, 1

Schmitz, F., \& Fleck, B. 1992, A\&A, 260, 447

Schrijver, C. J., DeRosa, M. L., \& Title, A. M. 2010, ApJ, 719, 1083

Shelyag, S., Erdélyi, R., \& Thompson, M. J. 2006, ApJ, 651, 576

Shelyag, S., Mathioudakis, M., Keenan, F. P., \& Jess, D. B. 2010, A\&A, 515, A107

Shelyag, S., Zharkov, S., Fedun, V., Erdélyi, R., \& Thompson, M. J. 2009, A\&A, 501,735

Singh, R. N. 1992, Ap\&SS, 191, 125

Sobotka, M., Brandt, P. N., \& Simon, G. W. 1997a, A\&A, 328, 682

Sobotka, M., Brandt, P. N., \& Simon, G. W. 1997b, A\&A, 328, 689

Sobotka, M., \& Hanslmeier, A. 2005, A\&A, 442, 323

Stangalini, M., Del Moro, D., Berrilli, F., \& Jefferies, S. M. 2011, A\&A, 534, A65

Thomas, J. H. 1985, Aust. J. Phys., 38, 811

Torrence, C., \& Compo, G. P. 1998, Bull. Am. Meteorol. Soc., 79, 61

Tritschler, A., \& Schmidt, W. 2002, A\&A, 388, 1048

van de Hulst, H. C. 1950, Bull. Astron. Soc. Neth., 11, 150

Van Doorsselaere, T., Ruderman, M. S., \& Robertson, D. 2008, A\&A, 485,849

Vernazza, J. E., Avrett, E. H., \& Loeser, R. 1981, ApJS, 45, 635

Verth, G., Erdélyi, R., \& Jess, D. B. 2008, ApJ, 687, L45

Wöger, F., von der Lühe, O., \& Reardon, K. 2008, A\&A, 488, 375 